\title{
Overview of the application of geospatial technology to groundwater potential mapping in Nigeria
}

\begin{abstract}
Sustainable development of groundwater resources in many developing nations like Nigeria is hampered by paucity of information about the resource. Geospatial technology has provided useful information in understanding the distribution of groundwater throughout the world for the purpose of planning, exploration, monitoring and conservation. Numerous studies have integrated remote sensing (RS) and geographical information system (GIS) in the assessment of groundwater potential in different parts of Nigeria. This paper therefore aims to review the literatures and reveal the successes and most importantly the challenges faced by such studies and provide useful recommendations for improvement in future research. A global perspective of advances in the application of geospatial technology to groundwater mapping was first captured from where the strength and weaknesses of the same application in Nigeria in terms of methodology of data extraction, modelling and validation were assessed. The review shows that less than 20 bibliographies are available with about $50 \%$ from the south-western part of the country. Few parameters controlling groundwater are often analysed and categorised without any standard. Most models are produced from software packages that do not allow weighting of thematic layers hence cannot incorporate expert judgements. Areas needing urgent attention for improvement include: time of remote sensing data acquisition, methods of parameter extractions, standard GIS software packages used, novel models and comparison between models and the need for model and map validations. Recommendations on country wide coverage of groundwater potential assessment, provision of groundwater monitoring facilities and strict adherence to groundwater development standards were also made.
\end{abstract}

Keyword: GIS; Groundwater; Mapping; Nigeria; Remote sensing; Review 